\title{
Adaptive Information Processing and a Systemic Biopsychosocial Model
}

\author{
Anthony J. Cotraccia \\ Private Practice, Ithaca, NY
}

\begin{abstract}
Shapiro's (2001) adaptive information processing (AIP) model portrays an innate healing system hypothesized to be composed of neurophysiological mechanisms of action causally related to the resolution of disturbing life experiences. The author expands the model to include psychosocial mechanisms and suggests that a model of a biopsychosocial system can best depict causal properties related to positive outcomes of eye movement desensitization and reprocessing (EMDR). Teleofunctionalist and evolutionary perspectives are applied: the first, to explain the inclusion of the psychological and social features highlighted in the updated model; the second, to support the hypothesis that AIP is a goal of the human attachment system. It is posited that bonding, following a disturbing life experience, facilitates the access of information related to previous states, thus allowing an update of self/world models. These interactions are analogous to psychotherapeutic encounters, with multiple levels of information processing at subpersonal, personal, and interpersonal levels. Analysis of the causal properties of personal and interpersonal levels supports a broader understanding of AIP's scope in conceptualizing psychopathology and informing treatment applications and research.
\end{abstract}

Keywords: EMDR; adaptive information processing; biopsychosocial; teleofunctionalism; internal working models

$\mathrm{O}$ riginally conceived to describe well-documented treatment effects of eye movement desensitization and reprocessing (EMDR) in thetreatmentofpost-traumaticstressdisorder(PTSD), Shapiro's(2001)adaptiveinformationprocessing(AIP) model has been described as a "working hypothesis" intended to start the model building process (p. 30). Themodelisbasedon"neurophysiological"structures comprisingan"innatehealingsystem"(Shapiro,2001, p. 30). Imbalances in the system are considered to be causedbytraumaticmemoriesandcontributetosymptoms of psychopathology (Shapiro, 2001).Processed memorynetworksareseenasthebasisforhealth(Shapiro, 2001, p. 32).

Thisarticlerecommendsenhancing themodelby including imbalances in personal and interpersonal processes as additional effects of disturbing life experiencesandviewing theirrestorationasadditional signs of well-being. Imbalances are conceptualized asproblemsinpersonalandinterpersonalcommunication and representation. This emphasis views the biopsychosocial context as a source of both trauma and healing. Consequently, the enhancement of communicationandrepresentationonalllevelsispresented as causally involved in AIP at the subpersonal level and therefore critical to effective EMDR.

\section{Information Processing Models} and Systems

A communication theory perspective on memory and psychopathology was speculated by the American mathematician Norbert Wiener (1948). The founderof "cybernetics,"Wienerconsideredtherole that information plays in both machines and natural systems. He noted that psychopathology was likely caused bythefunctionalimpairmentsresultingfrom structural problemsinthenervoussystem.Heconjectured that the challengeforthetreatmentofpsychopathologywould beto surmount thefact thatunlike withmachines, memoryin humansbecomespermanentthroughoutthe "systems"lifeand thereforethe system cannot be "reset." He observed that clinical applicationsofamodelfocusedonmemoryand psychopathologyatthetimeofhiswritingincludedfrontallobotomies(toremovethememoryphysically)and 
electroshocktherapy (tojarthecircuitsintonewpatternsoffiring).Suchlinearinterventionsseemtobelie themodeltheywerebuilton.Cyberneticsbroughtto lightthecausal propertiesrelated toinformation processingandfeedbackmechanismsthatdonotrelyon forces alone to determine the course of the system.

Central to the discourse of information processingmodelsisdefiningthesystemanddeterminingits boundaries.Theauthor'sexperienceintheclinicalapplication ofEMDRand systemic family therapy is the basisandmaininspirationforbroadeningtheboundariestoincludepsychologicalandsocialcomponents.

Thebiopsychosocial AIP model is conceptualized as a "nearly decomposable system" (Simon, as cited in Juarrero, 1999) which means thateach level maintains a distinct separation from the next while all are connected via feedback loops. It can also be considereda"hierarchicaldynamicsystem" wheretoplevels constrain the lower (Juarrero). These attributes of complex systems contribute to the proposed causal relationshipbetweenhigher(personalandinterpersonal) and lower (subpersonal) levels.

Thephysicalflow ofinformation betweencomponent parts makes the biopsychosocial AIP system an "informational system" (Juarrero, 1999). Seen from this perspective, thepathogenicnature ofdisturbing lifeexperiencesliesintheircapacitytodisruptcommunication and representation atanylevel.Information flowing within and between levels makes the system "vulnerabletonoiseandsensitivetocontext"(Juarrero, p.114).Theopenandrelativelyunequivocalexchange of information between and within levels of biopsychosocialAIPenhancestheaccumulationofpaststates ofadaptiveactions thathavelead to mutually beneficial outcomes for person and environment. System complexityisseenasenhanced behavioralflexibility.

Theauthorsuggeststhatthereisampleevidenceinthe literature on EMDR, trauma, philosophy of mind, and attachmenttobuildamodel of such asystem.InEMDR, theAIPsystemisthoughttoallowforlinkstoneuralnetworksthatpromotelife-preserving responsestostress thatbecomeintegratedintoanadaptiveemotionaland cognitiveschema(Shapiro,2001).Theincorporation of adaptivelyprocessedmemoriesintoapositiveschemaof the self/world is thought to bean outcome of effective EMDR in addition to the amelioration of PTSD symptoms (Shapiro, 2001). Thus, the survival value of using social engagement to deal with stress (Porges, as cited in Ogden, Minton, \& Pain, 2006) amplifies the salience ofhavingsophisticatedresourcesforsocialcognitionin coping responses to disturbing life experiences.

A biopsychosocial AIP model provides explanatory strength to EMDR case conceptualization and treatment planning by including the social context of theclient,thetherapist,andthetherapy.Thelocation of theinformationprocessingsystemwithinand without theclientassistsinconceptualizingrelevantrelational aspectsofpsychotherapyaswellasthesocialenvironmentwithinwhichtreatmenttakesplace.Suchamodel maybeableto systematicallyaccountformany of the variousfactorsthoughttobeinvolvedinsuccessfulpsychotherapy as described by Norcross (2007). As with thebehaviorofallcomplexnaturalsystems, treatment response is understood in retrospect (Juarrero, 1999) withacautionagainstdefinitiveprediction, insteadfavoring probabilisticassertionspronetochangeasthis multilevelcomplexinformationalsystemorganizesand reorganizes(Juarrero).Eachclient'sstoryprovidesthe necessarycontextfortreatmentplanning whilechance "natural occurrences" may also play a critical role in treatment outcome. Expanding the scope of the AIP systememphasizesthecriticalrolethattheavailability ofadaptiveinformationwithinthesystematlargeplays in the efficacy of EMDR treatment.

\section{Internal Working Models of Self}

Empirical evidence for a biopsychosocial AIP system is found in the role that memory appears to play in behavioralintegrationand social cognition. The construction of internal working models of self (IWMs; Bowlby, 1969)organized tocontrolbehaviorinvolves autobiographicalmemoryandpersonalandsocialinformationprocessing.Forhumans, theinternalization of our social history involves the brain, the mind, and the relationships (Panksepp, 1998; Siegel, 1999). The development of autobiographical memory systems gave humans the capacity to retrieve past states and bringthemforwardintime. Theresulting "autonoetic consciousness" (Tulving, 2000) or "extended consciousness" (Damasio, 1999) allows us to experience ourselvesintime.Wecandirectattentiontopast,present,andanticipatedfuturestatesandmentallysimulate possibleselvesand possibleworldsaswellaspossible relationships between them (Metzinger, 2003). Of particular importance to AIP is the internalization of examplesofcommunicating withcaregiversandconstructingadaptivestoriesofhowtodeal withstressful situationsencounteredacrossallbehavioralsystems.

In other words, IWMs constrain the attention and behaviorofbothcaregiverandchild.Thecapacityfor intrapersonal attunement (Siegel, 2007) of the caregiverand interpersonal attunement with thechild is "passed down." The child can then establish the capacity to form an open personal and interpersonal communicationchannel, relativelyfreefrom"noise"; 
this facilitates the entrainment of adaptive memory useful for the maintenance of the self/world boundary that will be necessary to integrate disturbing life experiences in the future.

Theauthorproposesthattheseearlyrelationships with caregivers can be understood as "context-sensitive constraints" (Juarrero, 1999) that have causal propertiesthatstructuretheexperiencesofthechild, thusmakingsomeoutcomesmorelikelythanothers. Theseoutcomesincludeintegration of memoryinto a healthy IWM or segregation of memory into disintegrated IWMs (Liotti, 2006).

When unconscious IWMs underlie a healthy selfconcept, they can be consciously invoked to further shapethemorefixedsubpersonal patternsofbehavior and to promote self-regulation and behavioral coherence. When an IWM supports "mutual feedback" (Juarrero, 1999) between the internal model, somatosensorysystems, and otherpeople, intrapersonalandinterpersonalattunementiseffective, and theindividuallearnsappropriateself-regulationand behavioral integration that is coherent with theirsocial environment.

\section{Possible Mechanisms of Action in EMDR}

Basedonclinicalexperience,Shapiro(2001)hasattributedneurophysiologicalmechanismsofactiontothe AIP model. Most research on EMDR's mechanisms of actionhasinvestigatedthebiologicalandpsychological elementsinvolvedinproceduralsteps(e.g.,eyemovements) related to theresolution of traumaticmemory (Maxfield, 2008). Various related theories have been proposed. These include the role of working memory in memory desensitization (e.g., Maxfield, Melnyk, \& Hyman,2008)andinterhemisphericintegrationenhancing memory recall (e.g., Propper \& Christman, 2008). Neurobiologicalhypotheseshavebeenadvanced,for example,Bergmann(2008)hypothesizedthatalternatingbilateralstimulationstimulatesandrepairsthalamic activity and fosters neurobiological integration.

\section{Mechanisms of Action From Information Processing Perspectives}

Informationprocessingmodelsthatareseenasrelevant to Shapiro's (2001) AIP model include the transferappropriate processing model, the cortical reinstatement model, the parallel distributed/connectionistic model,andthethalamocortical-temporalbindingmodel (EMDR International Association, 2009). The transferappropriateprocessingmodelconsiderstheconditions presentatthetimeofencodingandretrieval relevantto theencodingofmemoryandthatmemoryperformance is enhanced when recollection occurs in the context within whichthecontentwasencoded(Tulving,2000). Thecorticalreinstatementmodelsuggeststhattheneurological correlates of episodicmemory retrieval differ according to the type of information contained in the recollectedepisode. Theretrieval of a particulartype of memory content will "reinstate" themental state presentatthetimeofencoding(Johnson\&Rugg,2007).The paralleldistributedprocessing(PDP)modeldescribesthe representationofinformationasdistributedthroughout thebrain;memoryandknowledgearenotstoredexplicitly but between many "units," and learning can occur withgradualchangesinconnectionstrengthwithexperience (Rumelhart \& McClelland, 1986). The PDP model stresses that many units process information through sendingandreceivingexcitatoryandinhibitorysignals in a particular environment that promotes such communication between units (Rumelhart \& McClelland). Finally, the thalamocortical-temporal binding model positsthatanintegrativehippocampalformationprocess linkstogethervariousneuronalassembliesestablishedat the time an event was perceived via the $40-\mathrm{Hzgamma}$ band activity of the thalamus (Bergmann, 2008).

To understand how EMDR facilitates AIP, a synthesis of observations related to the interaction of neurobiologicalandpsychologicallevelsofprocessing provides the clearest picture. AIP seems to facilitate communication in suchawaythatautobiographical memorycanbeadaptivelyrepresentedandintegrated. Structuresinvolving basiccomponents ofa communicationsystemincludingachannel, distributeddata structures (sources of information), and an optimal environmentforthetransmissionandrepresentation ofinformation seemsalientto AIP.Thebiopsychosocialmodelsuggestssuchstructuresexiston personal andinterpersonallevelsaswellandhaveacausal relationshiptoeachlevelandultimatelyontheresolution of disturbing life experiences in EMDR treatment.

\section{A Historical, Contextual, and Temporal Biopsychosocial AIP System}

Dynamicalsystemstheoryasappliedtocomplexliving systems can help the EMDR clinician and researcher understandhowpsychologicaland social processes can be causally related to AIP. Tothis end, the author hasreviewedsomerelevantconceptstobegintheintegration of dynamical systems theory with AIP.

Causality

The first concept is that of causality. The exploration oftheinanimatephysicalworldhasled toproductive notionsof "whatcausesthingstohappen."However, 
naturalsystemsseemtooperateunderdifferentrules. Intheinanimateworld, itislogicaltoconsiderseparate entitiesexertingaforceononeanother.Inthatworld, the history of the entity is irrelevant to its course.

Whenconsideringlivingsystems, contextand time mustbebroughtintothescientificexploration ofcausality(Juarrero,1999).EMDRcliniciansconceptualizing acaseinAIP termsarepresented withexcellentexamplesofnaturalsystemsadaptingtotheirenvironments over time. To understand why natural living wholes dowhat theydo, weneed toconsidertheinitial conditionsofasystem, whatgoalitisorganizedaround, and how its history has shaped its course over time.

Clients are living systems embedded in their environments and they exchange information with it. Theconceptofnonlinearcausalitypositsthataliving system is its own cause because it uses its history to determinethesetofpossibleactionsatanyparticular momentintime (Juarrero, 1999). In contrast to force, context-sensitive constraint is understood to be the way that history shapes behavior.

Inaclosedsystemwhereinformationisnotshared between parts,onepart "causes" anothertodosomething, and the history of the latter is inconsequential because the system is governed by an overarching physical law (Juarrero, 1999), in which change does notoccur; thetrajectoryofanearequilibrium system is fixed. This type of system moves toward entropy, anditstrajectorycanbeexplained bytraditionalthermodynamic laws. Natural systems, however, are systemsthatcharacteristicallyresideatstatesfarfrom equilibrium. Such "nonlinear" systems are dynamic andparticularlysensitivetotheinitialconditionspresent at the time of their formation. Although initial conditionsarenotcauses, orforcesacting on thesystem, theycanhavesubstantialeffectsonthesystem's trajectoryovertime (Murphy\&Brown,2007).Clients bringboththeirgeneticandautobiographicalhistory forward in time to shape behavior in a dynamic interaction of their past, present, and potential future states with their environment. The current environment's evocation of memory constrains behavior leadingtostatesofincreasedordecreasedcomplexity in relationship to the present moment (Juarrero).

\section{Dynamic and Mutualistic}

Eachindividualisanextraordinarilysophisticatedsystem of systems (complex system) that uses self-representation to exploit autobiographical history to its fullest.Thiscomplexitycanbedescribedasdynamicand mutualistic.Abiopsychosocialinformationprocessing model describes how communication between the brain, mind, and world allows individuals to manipulate memory to create the experience of a self, world, a selfin a world, and the present moment (Metzinger, 2003). Thiscapacityforcomplexinformation processing provides the ability to simulate past, present, and anticipatedfutureexperiences,makingbehaviorallyrelevantinformationaboutselfandworldunconsciously and consciously available (Metzinger, 2003).

EMDR clinicians have witnessed and documented how the adaptive resolution of autobiographical memoryrelatestooverall behavioral integration beyond the alleviation of PTSD symptoms (Shapiro, 2001). The author believes an outcome of effective EMDR treatment is the enhancement of biopsychosocialcommunicationandrepresentationthatallows for the simulation of a phenomenal first person perspectiveandsupportsoptimalbehavioralintegration within a social context when confronted with astressor. An informational system's physical operations are organized around the exchange of information between parts and levels (Juarrero, 1999). EMDR therapists have been in a unique position to observe such information exchange at multiple levels and within social groups.

\section{Memory and Moving Around in Time and Space}

\section{Consciousness and Behavioral Integration}

It is well understood that individuals use memory to make their way through the spatiotemporal world. Theauthorbelievesthatadaptivelyprocessedmemories seem to enhance functioning in systems related to the sophisticated way humans intentionally move throughspaceandtime.Expandedlevelsofconsciousnessmakeintentionalmovementpossible(Metzinger, 2003, p. 60) via autobiographical memory that allows for the experience of self as a historical persona. Dworkin(2005)hasdescribedresourcesnecessaryfor successful EMDR treatment: attunement, mindfulness, andresponseflexibility.Adaptivelyprocessedautobiographicalmemoriesareintimatelylinkedtothese metacognitiveproperties. Theyprovidefunctionsnecessary to organize behavior in a spatiotemporal phenomenologicalworld.Objectsofattentionalprocessing andcognition (whichincludememory)arealwaysalso constituents of behavioral space (Metzinger, 2003).

Autonoeticconsciousnessor"mentaltimetravel" is a temporal process that organizes experience and differentiatesbetweenwhathashappened, ishappening, andmayhappen. Responseflexibilityreferstothe abilitytointentionallydelayaction.Togetherthesecapacitiesallowforamoresophisticatedconsideration 
of possible adaptive actions. In a neurobiologically integrativeclimate,memoriescanbeprocessedsimilarlytoexternalobjectsandbecomepartofconscious experience(Damasio,1999).EMDRmaybeconceptualizedasatypeofcoregulatedmentalsimulation that involvesaccessingmemorytointernalizeavariety of adaptivewayswecanmovein thespacesweoccupy.

\section{Establishing Reliable Biopsychosocial Communication}

Embedded but Separate:

A Need to Make Contact

Theindividual'scapacitytoexperienceselfasseparate fromanenvironmentinwhichsheisdeeplyembedded promotesbehavioralflexibility.Thisphenomenallyexperienced boundarybetween the bodyand theenvironmentemergesfrom thecommunicationbetween the brain, mind, and relationships (Metzinger, 2003; Siegel, 1999). However, notall of thiscommunication andrepresentingisavailableineverydayconsciousexperience.Infact,theselfandworlddirectlyexperienced bytheindividualarethemselvesrepresentationsbased onestimations. Individualsareironically unabletodirectly experience "ourselves" or the "external" world. Thisplacesapremiumonthenervoussystem'sability totransmit(communicate)andmanipulate(represent) information from sensory and memory systems.

Referredtoas"autoepistemicclosure"byphilosophy ofmind philosopherMetzinger(2003), humansarein apredicamenthedescribesasa"structurallyanchored deficit in the capacity to gain knowledge about oneself" (p. 32). The process of representing self in the world takes place at the subpersonal level and is not generallyavailableforconsciousreflection(Metzinger, 2003).Whileclientandtherapistmighttakesuch phenomenological experience for granted, it would be impossible to carry out the EMDR protocol without the experience of a personal boundary for the client.

\section{Subpersonal Communication and Representation}

Communication in the brain involves an exchange between particular structures of the brain and an external or internal stimulus. Gallistel and King (2009) developed thefollowingmodeltoexplainthis process. To make contact with an external stimulus, there is an interaction with sensory receptors that provideinformationaboutwhatishappeningoutside ofthebrain.Actingasanextremelycomplexsubpersonalprocessing center, thebrain reliesonextracting meaning from a wide variety of signals. This process of extractingmeaningfrominformationiscalledrepresentation.Inorderforcommunicationtotakeplace at this level, a neurobiological channel is needed to carrythe"spikes,"whicharetransmutedexternalsignals produced by sensory receptors. A "spike train" comprisesthesubpersonalchannelandincludesneurons, which facilitates the transmission of information and allows the brain to extractinformation from these signals. Information removed may be used to inform present behaviororcontained forlateruse to influencefutureactions.Suchachannelprovidesthe necessaryorganizationalstructureasystemneedsto effectively communicate, and therefore, represent.

Personal and Interpersonal Communication and Representation in AIP

When phenomenalexperienceispossible,clientand therapistareinapositiontoreflectonrepresentations of memory networks containing related to the presenting complaints. Theauthorbelievesthatintrapersonalattunement(Siegel,2007)isthecommunication channeloperatingatthepersonalandinterpersonal levels through which this process occurs. It has the function ofensuringtherelativelyunequivocaltransmissionofinformationrisingupfrom thesubpersonal level.Throughintrospection, theclientcanbecomeattunedtohisorherbiopsychosocialstateandthepair's capacitytoguidetheirattentionmaximizesinformationextractionrelevanttorepresentingdysfunctional networks as directed in the EMDR protocol.

\section{Trauma and Noise Reduction}

There is a vulnerability to noise within any complex system.Noiseisanythingthatinterfereswithinformation-carryingsignalsreachingtheirintendedreceiver (Gallistel\&King,2009;Juarrero, 1999;Shannon, 1948). Based on the current EMDR literature and information theory, trauma may be conceptualized from an informational theoretic perspectiveasnoisethat disrupts communication at all levels of AIP. The manner in which trauma disrupts communication between episodic and semantic memory systems in rapid eye movement (REM) stages of sleep would be a subpersonal example of "trauma as noise." The loss of communication between body and mind in somatoform dissociation(Nijenhuis,2004)would beanexampleon thepersonallevel,andkeepingsecretsaboutabuseina familywouldbeanexampleontheinterpersonallevel. Intheseexamples, trauma becomes defined moreby thelackofresourcesforattunementandcommunication ratherthan bythenatureofthestimuliasemphasized by Freud and Reik (Reik, 1945). 
The assumption that AIP at higher levels of functioning is causally related to the subpersonal level leadstotwopropositions ofthisarticle.Oneisthatthe reductionofnoiseandrelativelyunequivocalcommunicationbetweenelementsofthesystematall levelsis criticaltoadaptiveprocessing.Thesecondproposition isthatstructuresrelatedtointerpersonaland personal informationprocessingarecomponentpartsoftheAIP systemtotheextenttowhichtheyreducenoiseandallow for reflection on mental content relevant to the subpersonal memory networks being targeted. This makes thebiopsychosocial AIP system an integrated setofmultilayeredstructuralisomorphiesbuiltonthe subpersonallevelofprocessingandorganizedaround communication and representation.

\section{The Subjectivity of Information Processing}

Shannon's (1948) information theory suggests that the extent to which a receiver has narrowed down a broad range of possible values for a stimulus is the extent to which the receiver has had an "informative experience" (Gallistel \& King, 2009, p. 6). This situation highlights the subjectivenature ofinformation. The availability of possible states of self and world in autobiographicalmemoryallowsonetodistinguish between potentially infinite amounts of online sensory input. This makes the accumulation of memory important not only for constructing adaptive mental models that promote attunement but also for fine-tuned perception of differentiated states of self and world.

A dynamic model of self that allows for an ever expanding IWM will therefore increase a client's capacityforaccuratesimulationsofreality.Likewise, an expansiveand expanding IWM of the clinician is also valuable. From an information theoretical perspective, the clinician's prior knowledge of the client's biopsychosocial state increases the probability that client's messages will be information-bearing messages. A clinician will be unable to assist the client in distinguishingthemeaningofhisorhervariousstate to his or her biopsychosocial situation if the clinician has no prior awareness that a given possible state of the world might exist.

\section{Social Attunement}

Because of the presumed causal properties of social states, theauthorexpanded theconceptsofintrapersonaland interpersonal attunement(Siegel,2007) to includesocialstates.Eachclientexistsatanygivenhistoricalmomentwithina particularsocial context that shapeshisorherphenomenal experience. The social state oftheclientisacondition that is causally related to AIP by promoting or thwarting attunement. At the interpersonallevel,socialattunementiscriticaltopositive outcomes in psychotherapy. For instance, clinicalexperiencewithEMDRsuggeststhattheclinician's awarenessoftheparticularclinicalissuesfacingtheclient (e.g., age, family history, sexism, racism) is critical to successful EMDR treatment (Shapiro, 2001).

The author believes that when biopsychosocial attunement is attained, the "state of co-regulation" (Dworkin,2005)maintainedisthephysicalrealization of a channel of interpersonal communication. This allows the representation of information and construction ofrepresentations oftheselfand world that assist the therapist in directing attention to relevant aspects of the biopsychosocial experience of the clientthatallowforthetargeting of salientsubpersonal networks of memory.

\section{Representational Structures That Structure the AIP System}

\section{Mental Models and States of Mind as Context Sensitive Constraints}

Shapiro (2006) describes the AIP system as an "innate healing system forged over millions of years" (p. 5). As implied previously along with biological structures, temporal,andsocialstructuresareamong the evolutionary tools humans have obtained to maximizetheadaptivebehavioralresponsestoself/ worldsituations."Mentalstatespossesscausalproperties, which, in acertaingroup of personasorunder theselectivepressureofaparticularbiologicalenvironment, can bemoreorlessadequate" (Metzinger, 2003, p. 26).

The existence of any biological tool implies a functionalrelationshipbetweenthetoolandthesystem/organism using it (Metzinger, 2003). States of mindandtheircorrespondingself-modelshavebeen optimized through their causal relationship to neurobiological structures over time. The presence of sophisticatedand complexmental contentfound in representationalsystemsseemsto bethemostplausible explanation for the capacity of social cognition thatallowsustopayattentiontotheconcepts, behaviors, and mental models of others (Metzinger, 2003). The author believes that mental states that facilitate AIP are those that allow an individual to reflect on his or her self/world models making himself or herself, his or her concepts, and his or her behaviors the object of his or her own attention and the attention ofatrusted other.Inthis way, thesebecomecontextsensitive constraints related to AIP. 
Reliablebiopsychosocialcommunicationestablishes a context within which mental models in need offurtherupdatingcanbeidentifiedviacorrespondingstates of mind reflected on in a trusting relationship. This ability to representselfto enhanceself-organization emergesinthecontextofrelationship.Thisinherency of the social in the personal is logical for an organism thatthrivesinattunedinterpersonalrelationshipsand it is emphasized in the model of AIP.

Safety, Responsibility, and Choices: Meaning Structures of a Biopsychosocial AIP System

The "engine" of a biopsychosocial AIP system is those structures that promote the relatively unequivocal transmission of messages on both the sending and receiving end. The author believes that the EMDR traditionhasidentifiedatriadofphenomenalcontentfound inIWMsthataidsinstructuringthebiopsychosocialAIP system.Positivecognitionsrelatedtosafety, responsibility, andchoicehavelongbeendocumentedintheEMDR literature(Shapiro,2001)asemergentphenomenalcontentindicativeofthepresenceofadaptivelyprocessed autobiographicalmemory.Intheirnegativeform,they indicatethepresenceofunprocessedmemory.Forexample,thenegativecognitionsassociated withtargeted memories in EMDR have been distilled down to "I'm not safe," "I'm to blame," and/or "I'm helpless." It is postulated that the inability to incorporate an experienceintoahealthyself-conceptseemstoberelatedprocessesassociated with threattolife,experiencing toxic shame,and/orfeelingtrapped.Alloftheseexperiences maketheindividualvulnerabletoaffectdysregulation and distortions of time, place, and person.

Fear, shame, and rage are innate stimulus bound affective responses to such situations (Panksepp, 1998). Extreme levels of these emotions can inhibit information processing in the moment on the subpersonal, personal, andinterpersonallevels(e.g.,the role of shame in dissociative responses). Examples from psychotherapy of how a lack of safety, undue responsibility, and/or lack of choice affect communication, and therefore, adaptive representations of experiencethatfacilitateinformationprocessinghave been described by Dworkin (2005). Fear, shame, and helplessnesscanemergeasaresultofintersubjective interactionsduring psychotherapyandcanstifleAIP. When the EMDR therapist pays attention to these emotionalstatesintheclientandfacilitatesareturnto a state of "co-regulation," AIP can resume. Dworkin hassuggestedthatrepairingtheruptureofsuchstates is imperative to successful EMDR treatment.
The author supports Dworkin's (2005) assertion and hypothesizes that when such a rupture is interpersonally processed in a session as described in the "relationalinterweave"(p.39)intervention, thebiopsychosocial AIP systemitselfisstrengthened.Inother words, theactual relationshipand theupdatedIWMs structure the bio-psychosocial AIP system and are thoughttobecausallyrelated totheadaptiveresolution of the target memory network. When therapist and client create a context within which it is acceptable to feel safe and focus on the target experience/ memory, acceptabletoowntheexperience/memory, and acceptable to use the experience/memory for current and future simulations, AIP is strengthened.

\section{"Optimal" Internal Working Models and a Healthy Self-Concept}

Self-directedattunementandintrospectiondependon theindividual's ability to directhis ownattention. This capacity is called attentional agency (Juarrero, 1999; Metzinger,2009).IWMscontainingarobustsetofprior probabilitiessuggestingthattheindividualissafe,can own experiences, and has more choice will support the regulation of fear, shame, and rage allowing for attentionalagencyand ongoing communication and representationofwhatishappening.Theauthorhypothesizesthat"optimalinternalworkingmodelsofselfand world" promoteafeeling of safetybyallowingtheindividualtoownthedisturbingexperienceandtoproblem solvehowtorepresentandremedyit, inamannerthat benefitsselfandenvironment.Inotherwords, thisprocessisaccomplishedinpartthroughthemaintenance of a self/world boundary. The specific self-conceptual triadrelatedtosafety, responsibility, andchoiceswould beconsideredlinguisticrepresentationsofsuchmodels hypothesizedtobeprevalentamongindividualswitha secure/autonomous attachment status.

\section{Goals of the Biopsychosocial AIP System}

The use of both fixed action patterns and learned behaviors is prevalent among more complex species (Murphy \& Brown, 2007). Behavioral flexibility is maximized via optimal IWMs. Bowlby's (1969) "environmentofevolutionaryadaptedness" describesthe emergence of innate "stimulus bound" unreflective behaviorsshaped byevolutionthatprepareanorganismforlifeinaspecificsetting.Therearealsocharacteristicbehavioralresponsesgeneticallyencodedwithin thecentralnervoussystemofhumansandothermammals related to theabsence of certain environmental stimuli that are salient to survival (Bowlby, 1969; Panksepp, 1998).Theauthorbelievesthatanattuned 
interpersonalrelationshipistheenvironmenttowhich an infant's fixed patterns of attachment behavior are designedtoanticipateandrespond.Furthermore, the guaranteethattheinfantwillconsistentlyseekandfind attunementishypothesized tobefoundinthecapacity to developIWMs that selectivelyentrainattention and memories that maximize attunement. Thereby ensuringattentionalagencyandadaptivebehavioral integration willoccurviatheconstruction ofaccurate representations of self and world.

\section{How a Biopsychosocial AIP System Is Organized and Maintained}

Theauthorhypothesizesthatthepresenceofattunement in the environment acts as the initial condition that an AIP system organizes around. Furthermore, IWMscanbeseenas datastructurescontaining information usefulto depict what dynamic systemstheorists call attractors. An attractor is an abstract shape thatdelineatesthestatespaceovertimewithinwhich anygivensystemresides(Juarrero, 1999, p.152;Murphy \& Brown, 2007, pp. 75-76). They represent the multitudeofcontext-sensitiveconstraintsthatendure and influence the probability that a system will behave with predictable patterns (Juarrero). An attractor also represents the space a system will return to if perturbed outsideofitstypical pattern of behavior. Forinstance,theconceptualization of humanbehaviorasresultingfromasystem'spredictabletrajectory throughstatespacehasbeenused byPutnam(1997) to describe dissociative disorders.

TheauthorbelievesthatIWMsactasattractorsthat shapeanindividual'strajectory toward the developmentofanAIPsystem.Inaddition, theestablishment of a biopsychosocial AIP system may be the apex of theattachmentsysteminasecureautonomousindividual. He has found the conceptualization of IWMs as data structures containing information useful in describingsalientattractorsofabiopsychosocialAIP systemasausefuldiagnostictoolin determining the overall tendency for an individual to respond with attunementinanygivenstressfulsituation. Byextension, theassessmentofthecapacityforintrapersonal andinterpersonalattunementhasalsobeenusefulin the author's clinical workto assess the robustness of the biopsychosocial AIP system itself.

\section{Discussion}

AphylogeneticperspectiveofAIPsuggeststhatemergentpropertiesofacomplexnaturalsystemthatuses its history to promote behavioral flexibility are caus- allyrelatedtoadaptiveprocessingofautobiographical memory.Theauthorpresentsthetheoreticalperspective of a biopsychosocial AIP system to capture the waythatpersonalandinterpersonalprocesses(mental and social states) may be causally related to the adaptive resolution of disturbing life experience. In particular,advancesintheunderstanding ofcomplex naturalsystemsandtheircapacityforsubjectivityand intentional actions have offered important insights that can beintegrated with the existing neurophysiological model of AIP.

In addition, current trends in EMDR have focused on relational aspects of AIP (Dworkin, 2005;Dworkin \&Errebo,2010)ascliniciansandresearchersadaptthe protocoltotreatmorecomplexpsychiatricconditions. These adaptations have often led to the integration of EMDR and attachment theory. This article has moved thediscussionfrom theclinicalintegration of EMDR and attachment theory to the theoretical integration of the two. Focusing merely on traumatic memory in a linear fashion as if it were a "germ" that "causeslikeaforce," betrays the deeperunderstandingsofhowdisturbinglifeexperiencesand thestateof abiopsychosocialAIPsystem relatetooneanotherin determiningthetrajectoryofpsychopathologywhere memory is concerned.

What happens when an infant develops in an environmentwhereattunementisnotprevalentorpossible? Informationalclosure(Juarrero, 1999)describesthetenacitywithwhichasystemmaintainsitsintegritydespite exchangeswiththeenvironment. Theauthorsuggests that a systemic understanding of AIP is necessary to avoid clinical errors and frustration in the therapeutic alliance when an individual does nothave the capacity forattentionalagencyrequiredtoprocessdisturbingexperiences with EMDR. The biopsychosocial AIP model offersmethodologicalinsightsderivedfromconsidering thedynamics of suchasystem.Spacelimitations of this articleanditsfocusontheoryrequireafollow-uppaper to adequately address these insights.

Oneinsightis thevalue of themodelinidentifying the extent to which a client is embedded in an AIP system. If adaptively processed memories function withinasystemto bring informationforwardintime, tooptimizethesimulationandactualizationofbehaviorsthatmaximizethefitamonganindividualand his or her environment, then the use of such adaptive memoriesoughttobeinferredfromcoherentbehavioral functioning. Likewise, the absence of adaptive processing ought to be inferred from disorganized behavioral repertoires.Itwouldalsobesound toconsider that the more AIP "like" a system is, the "more 
conscious" the individual will be and the more he or she will behave more "intentionally."

ThebiopsychosocialAIPmodelcanenhancetreatment planning in the following ways: The client's maladaptive attractors can be described and identified by the range of negative cognitions and affects associated with recent triggers and past touchstone memories. These representations of IWMs can be plottedonachartwithacorrespondingdifferentiallist ofassociatedreflexivebehaviorstargetedforchange. Suchanexploration willyield thegenericelementsof situations likely to move the system into the closed state. Becausebehaviors of complexnatural systems are multiply realizable, this approach to treatment planning can assist in making sense of how the array of maladaptive behaviors in the client profile are organizedaround specificself/world interactions.Alist ofadaptiveself-representationsandreflectiveactions tailored to specificclientsituationscanalsobeidentifiedearlyintreatmenttohighlightwhereskillbuilding needs to take place as updates to IWMs are made.

Finally, the author believes that a biopsychosocialmodel supportsthelong-held adage ofFrancine Shapiro, (2009) that EMDR begins when the client walks in the door. Beginning with step one in the protocol, the pair construct macro representations of memory networks by maintaining biopsychosocialattunementandrecordinginformation related to areas ofinhibitiontogrowth.Theserepresentations, whetherverbalorwritten, guidethepair'sattention. The use of the EMDR self-report scales and body scansalsoconstrainthepersonalandsubpersonallevels of the client. Infact, the personaland subpersonal experiences of the therapist become constrained as well, and ifthereis minimalnoise interfering with the therapist's intrapersonal attunement, the dyad will maintain a state of coregulation necessary for AIP. The author hypothesizes that these are examples of howinterpersonalAIPisanemergentpropertyofthe biopsychosocial AIP system.

\section{References}

Bergmann, U. (2008). The neurobiology of EMDR: Exploringthethalamusandneuralintegration.Journal of EMDR Practice and Research, 2(4), 300-314.

Bowlby,J.(1969).Attachmentandloss:Vol.1.Attachment. New York: Basic Books.

Damasio, A. (1999). The feeling of what happens: Body andemotioninthemaking ofconsciousness.NewYork: Harcourt.

Dworkin, M. (2005). EMDR and the relational imperative. New York: Routledge.
Dworkin, M., \& Errebo, N. (2010). Rupture and repair in the EMDR client/clinician relationship: Now moments andmomentsofmeeting.JournalofEMDRPracticeand Research, 4(3), 113-123.

EMDR International Association. (2009, December). EMDRIA definition of EMDR. EMDRIA Newsletter.

Gallistel, C. R., \& King, A. P. (2009). Memory and the computational brain:Why cognitive science will transformneuroscience.Chichester,United Kingdom: Wiley-Blackwell.

Johnson, J. D., \& Rugg, M. D. (2007). Recollection and thereinstatementofencoding-related corticalactivity. Cerebral Cortex, 17(11), 2507-2515.

Juarrero,A.(1999).Dynamicsinaction:Intentionalbehavioras a complex system. Cambridge, MA: MIT Press.

Liotti, G. (2006). A model of dissociation based on attachment theory and research. Journal of Trauma and Dissociation, 7(4), 55-73.

Maxfield, L. (2008). Considering mechanisms of action in EMDR. Journal of EMDR Practice and Research, 2(4), 234-238.

Maxfield, L., Melnyk, W. T., \& Hayman, G. C. A. (2008). A working memory explanation for the effects of eye movements in EMDR. Journal of EMDR Practice and Research, 2(4), 247-261.

Metzinger,T.(2003).Beingnoone:Theself-modeltheoryof subjectivity. Cambridge, MA: MIT Press.

Metzinger,T.(2009).Theegotunnel:Thescienceofthemind and the myth of the self. New York: Basic Books.

Murphy, N., \& Brown, W. S. (2007). Did my neurons make medoit?Philosophicalandneurobiologicalperspectiveson moralreasoningandfreewill.NewYork:OxfordUniversity Press.

Nijenhuis, E. R. S. (2004). Somatoform dissociation: Phenomena, measurement, andtheoreticalissues.New York: Norton.

Norcross,J.C.(2007,September).Psychotherapyrelationships thatwork:Evidence-based practicesinEMDR.Paperpresentedattheannualmeeting oftheEMDRInternational Association, Dallas, TX.

Ogden, P., Minton, K., \& Pain, C. (2006). Trauma and the body:Asensorimotorapproachtopsychotherapy.NewYork: Norton.

Panksepp,J.(1998).Affectiveneuroscience:Thefoundationsof humanandanimalemotions. NewYork:OxfordUniversity Press.

Propper, R. E., \& Christman, S. D. (2008). Interhemispheric interaction and saccadic horizontal eye movements. Journal ofEMDR Practice and Research, 2(4), 269-281.

Putnam,F.W.(1997).Dissociation inchildren and adolescents:Adevelopmentalperspective.NewYork:Guilford Press.

Reik,T.(1945).Thecompulsiontoconfess:Onthepsychoanalysis of crime and punishment. New York: Grove Press.

Rumelhart, D. E., \& McClleland, J. L. (1986). Parallel distributedprocessing:Explorationsinthemicrostructureof 
cognition:Psychologicalandbiologicalmodels.Cambridge, MA: The MIT Press.

Shannon,C.E.(1948).Amathematicaltheoryofcommunication. TheBellSystem Technical Journal,27,379-423.

Shapiro,F.(2001).Eyemovementdesensitizationandreprocessing: Basicprinciples, protocols, and procedure(2nded.).New York: Guilford Press.

Shapiro,F.(2006).EMDR:Newnotesonadaptiveinformation processingwithcaseformulationprinciples,forms, scripts and worksheets, version 1.1. Watsonville, CA: EMDR Institute.

Shapiro,F.(2009,August).EMDRupdate:Theory, research, and practice.Paperpresentedattheannualconferenceof EMDR International Association, Atlanta, GA.
Siegel,D.(1999).Thedevelopingmind:Howrelationshipsand thebraininteracttoshapewhoweare.NewYork:Guilford Press.

Siegel,D.(2007).Themindfulbrain:Reflectionandattunement in the cultivation of well-being. New York: Norton.

Tulving,E.F.(2000).Theoxfordhandbookofmemory.Oxford: Oxford University Press.

Wiener,N.(1948).Cybernetics:Orcontrolandcommunication intheanimalandthemachine.Cambridge,MA:TheMIT Press.

Correspondenceregarding thisarticleshould bedirected to Anthony J. Cotraccia, 207 East Court Street, Ithaca, NY 14850.E-mail: cotracc@twcny.rr.com 